\title{
In vitro drug release rates from asymmetric-membrane tablet coatings: Prediction of phase-inversion dynamics
}

\author{
Sacide Alsoy Altinkaya*, Hacer Yenal \\ Izmir Institute of Technology, Department of Chemical Engineering, Gulbahce Koyu, 35437 Urla-Izmir, Turkey
}

Received 27 May 2005; received in revised form 29 September 2005; accepted 30 September 2005

\begin{abstract}
Most of the controlled-release systems developed for drug delivery applications depend on membrane technology. The dense structure of some membranes used in controlled-release systems can excessively prolong the release of drug due to the low permeability of the coating to drug. To increase the drug release rate, asymmetric-membrane tablet coatings were prepared by a phase-inversion technique using cellulose acetate/acetone/water solution. The roles of the composition of the membrane solution and the evaporation condition on the release rate of drug were determined using in vitro dissolution and morphological studies and predicted phase diagrams. Results show that drug release from asymmetricmembrane based tablet coatings is primarily governed by the dynamics of the phase-inversion process with zero-order or near-zero-order release easily achievable. In an attempt to derive an empirical expression for the release rate of drug as a function of composition of the coating solution, a statistical experimental design was used. Good fit of the experimental data by the empirical expression was obtained. In addition, the predictive capability of the model equation was also found to be satisfactory. Analysis of the significance of each term in the expression indicates that the cellulose acetate:acetone ratio has the most significant influence on the release rate of theophylline.
\end{abstract}

(c) 2005 Elsevier B.V. All rights reserved.

Keywords: Asymmetric-membrane; Phase-inversion; Statistical design; Cellulose acetate; Theophylline

\section{Introduction}

Different controlled-release systems were developed for drug delivery applications to maintain a drug level in the body within a specificed therapeutic window. This usually implies achieving prolonged, zero-order release rate over the desired duration of drug delivery. Many controlled-release systems in pharmaceutical industry rely on membrane technology in which a drugcontaining core is surrounded by a membrane, and the release rate of the drug is controlled by its diffusion through the membrane [1-5]. In addition to diffusional release, osmotic pumping mechanisms contribute to the total drug release rate, if either the drug is highly soluble or an osmotic agent is added to the active core [6-8]. The membrane coating used in conventional devices usually has a dense structure with a hole drilled through the coating through which the drug is delivered. In some cases, drug delivery ports are formed by adding leachable materials to the coating $[9,10]$. The release kinetics from dense membrane

\footnotetext{
* Corresponding author. Tel.: +90 2327506658.

E-mail address: sacidealsoy@iyte.edu.tr (S.A. Altinkaya).
}

coated drug delivery systems is controlled by: (1) the concentration difference and/or osmotic pressure difference across the membrane; (2) the permeability of the membrane to water and drug; (3) the thickness of the membrane. The main problem with these systems, in the absence of a hole, is an excessively prolonged drug release due to the low drug permeability of the coating. In an effort to increase the permeability of the coating, plasticizers and water-soluble additives were incorporated in the membrane forming solution and multilayer composite coatings were prepared $[9,11,12]$. Recently, the advantage of using asymmetric-membrane capsules for osmotic and transdermal delivery of drugs was illustrated by different research groups [6-8,13-17]. Compared to other conventional osmotic delivery devices, the permeabilities and the release rates of the asymmetric-membrane capsules/tablets were determined to be higher [6,7]. The structure of this type of membrane is characterized by a relatively thin, dense skin layer supported on a highly permeable, thicker and porous sublayer that provides mechanical strength and durability [8]. Herbig et al. [6] have shown that the outer surface of the asymmetric-membrane coatings is a key factor to control the drug release rates. 
Asymmetric-membranes are usually made by a phaseinversion technique in which an initially homogenous polymer solution is transformed to a porous, solidified structure by different external effects, such as thermal quenching or exposure to a nonsolvent bath. The morphology of the resulting membrane structure that controls the rate of drug release is strongly influenced by the phase-inversion dynamics. Thus, the ability to formulate efficient drug delivery systems based on the asymmetric-membrane coatings requires a detailed study of the effect of phase-inversion dynamics on the morphology of the membrane and the drug release behavior.

The goal of the studies in this paper is to demonstrate the relationship among the membrane preparation conditions, the resulting morphology and the drug release properties of the membranes. To achieve this goal, the in vitro release of a model compound, theophylline, from asymmetric-membrane tablet coatings is determined, and the morphology of the coatings is examined. In addition, the dynamics of the phase-inversion is quantified in terms of ternary phase diagrams coupled with composition paths determined from a mathematical model developed previously by our group [18]. To draw meaningful and objective conclusions from experimental data and derive an empirical expression for the release rate of drug, compositions of the coating solution are chosen using statistical experimental design.

\section{Materials and method}

Tablet cores were prepared by compressing the drug without any excipient using a hydraulic press operated at $110 \mathrm{MPa}$. Stainless steel die with a diameter of $1.2 \mathrm{~cm}$ was used to produce $400 \mathrm{mg}$ drug tablet cores. The model drug theophylline was supplied by Eczacibasi A.S. Asymmetric-membrane tablet coatings were applied using a dip coating process (Dip Coater Nima, type: D1L, serial no.: 327). Coating solutions were prepared by dissolving cellulose acetate (Aldrich) in a solution of acetone (Merck) and water. To eliminate variations in final coating thicknesses, the rate of withdrawal of the tablets from the solution was adjusted. Immediately after coating, tablets were rotated for even distribution of the viscous membrane solution, transferred into an environmental chamber (Angelantoni Industries, Italy, Challenge Series, model number: $\mathrm{CH} 250$ ) and kept there for $2 \mathrm{~h}$ to allow for evaporation of both the solvent (acetone) and nonsolvent (water). The temperature and relative humidity of air in the environmental chamber was adjusted as $25^{\circ} \mathrm{C}$ and $50 \%$, respectively. Tablets were allowed to dry further for a minimum of $24 \mathrm{~h}$ at room temperature prior to dissolution experiments. The release rate of theophylline from the tablets was determined by the United States Pharmacopeia (USP) XXIII dissolution methodology (rotating paddles, $50 \mathrm{rev} . / \mathrm{min}$; temperature, $37^{\circ} \mathrm{C}$; dissolution medium, $900 \mathrm{ml}$ ) using a dissolution tester (Caleva 10ST). To simulate the actual dissolution environment in the body, the $\mathrm{pH}$ of the dissolution medium was kept at 3 during the first $3.5 \mathrm{~h}$ by adding 8.5 vol.\% phosphoric acid to $900 \mathrm{ml}$ distilled water and then increased to 7.4 by adding $5.3 \mathrm{M} \mathrm{NaOH}$ to the dissolution medium and kept at this value until the end of the dissolution test. To determine the quantity of drug released from the tablets, samples were taken periodically and assayed by UV spectrophotometry (Shimadzu UV-1601) at a wavelength of $272 \mathrm{~nm}$. Dissolution experiments were performed on three tablets and the release profiles were reported as the arithmetic average of the three experimental runs. Morphology of the membranes was examined using scanning electron microscope (SEM) (Philips, XL-30SFEG). Samples were coated with gold palladium using a Magnetron Sputter Coating Instrument. The thickness of the dense skin layer, the overall porosity, and the average pore size were determined from image analysis of micrographs showing cross sections of the membranes.

\section{Statistical design of experiments}

To determine the influence of the composition of the coating solution on the release rate of drug, experiments were statistically designed using a commercial software package called Design-Expert [19]. The system studied in this paper consists of three components with compositional restrictions as shown in Eq. (1) below:

$5 \leq \omega_{1} \leq 15, \quad 70 \leq \omega_{2} \leq 90, \quad 5 \leq \omega_{3} \leq 15$

where $\omega_{i}$ is the wt.\% of component $i$ and 1-3 represent cellulose acetate, acetone, and water, respectively. Any composition outside these limits will probably fail to produce a successful asymmetric-membrane coating. In mixture experiments, the factors are the compositions of the mixture components, and the sum of the fractions of all components is equal to one. Therefore, the factor levels are mutually dependent. Thus, factorial experimental designs are not suitable for response surface modeling of mixtures since such designs require that the experimental treatment combinations be determined by independent adjustments of each component level. In addition, a standard response surface design cannot be used either due to the same constraints. Consequently, using the constraint levels shown in Eq. (1), a D-optimal design was generated by Design-Expert software package. The 14 experimental formulations determined are shown in Table 1. The lower and upper limits on the weight fraction of each component are required to: (a) obtain appropriate viscosity of the solution and coat the tablets uniformly and (b) induce phase separation, thus, forming a porous membrane structure. These constraints were established based on preliminary dissolution experiments, available literature data and the simulation results reported by Altinkaya and Ozbas [18].

Of the 14 formulations listed in Table 1 , six experimental runs were required to fit the quadratic mixture model, four additional distinct runs were used to check for the lack of fit and finally four runs were replicated to provide an estimate of pure error. DesignExpert used the vertices, the edge centers, the overall centroid and one point located halfway between the overall centroid and one of the edge centers as candidate points. Additionally, four vertices of the design region were used as check points [19]. 
Table 1

Release rates of theophylline from the asymmetric-membrane tablet coatings, the results of fitting the release profiles to zero-order kinetics, and the precipitation times determined from the model predictions

\begin{tabular}{|c|c|c|c|c|c|}
\hline \multicolumn{3}{|l|}{ Compositon (wt.\%) } & \multirow[t]{2}{*}{ Release rate $(\mathrm{mg} / \mathrm{min})$} & \multirow[t]{2}{*}{$R^{2}$} & \multirow[t]{2}{*}{ Precipitation time $^{\mathrm{a}}(\mathrm{s})$} \\
\hline Cellulose acetate & Acetone & Water & & & \\
\hline 15 & 80 & 5 & 0.036 & 0.9871 & $2671 / 2671$ \\
\hline 15 & 80 & 5 & 0.036 & 0.9864 & $2671 / 2671$ \\
\hline 5 & 90 & 5 & 0.45 & 0.9757 & $660 / 660$ \\
\hline 5 & 90 & 5 & 0.36 & 0.9864 & $660 / 660$ \\
\hline 15 & 70 & 15 & 0.036 & 0.9908 & $3374 / 3374$ \\
\hline 15 & 70 & 15 & 0.027 & 0.9925 & $3374 / 3374$ \\
\hline 5 & 80 & 15 & 0.36 & 0.9928 & $1000 / 1000$ \\
\hline 5 & 80 & 15 & 0.36 & 0.9876 & $1000 / 1000$ \\
\hline 5 & 85 & 10 & 0.27 & 0.9918 & $675 / 675$ \\
\hline 10 & 80 & 10 & 0.054 & 0.9958 & $1751 / 1751$ \\
\hline 15 & 75 & 10 & 0.054 & 0.9975 & $2314 / 2314$ \\
\hline 10 & 85 & 5 & 0.036 & 0.9887 & $2554 / 2554$ \\
\hline 12.5 & 77.5 & 10 & 0.036 & 0.9902 & - \\
\hline 10 & 75 & 15 & 0.063 & 0.9889 & $1484 / 1484$ \\
\hline
\end{tabular}

${ }^{a}$ The first number corresponds to the precipitation time at the tablet/coating interface.

\section{Determination of phase diagrams and composition paths}

Dynamics of the membrane formation process is predicted by combining the kinetics and thermodynamics of the system simultaneously. An appropriate thermodynamic model is necessary to construct the ternary phase diagram and to formulate the boundary conditions of the kinetic model. Phase separation is considered to occur when a mass transfer path touches the binodal curve. In this study, a robust algorithm developed previously by our group was used to construct the phase diagram [18]. The algorithm utilizes Flory Huggins thermodynamic theory with constant interaction parameters. The composition paths were determined from the kinetic model equations, which consist of coupled unsteady-state heat and mass transfer equations, film shrinkage, and complex boundary conditions. The details of both the thermodynamics and kinetic equations can be found in our previous study [18]. We have assumed that the kinetic model derived for a plane geometry can used to predict the membrane formation process on a tablet surface. This assumption is fairly reasonable since the thickness of the coating is very small, thus, the cylindrical geometry can be approximated as the plane geometry.

\section{Results and discussion}

\subsection{Effect of composition of the coating solution}

The effect of changing the composition of the casting solution is well documented for asymmetric-membranes prepared for separation applications [20-27]. However, there are relatively few quantitative studies illustrating the relationship between the composition of the casting solution and the drug release rate from the asymmetric-membrane coated tablets/capsules $[6,16]$. To investigate such a relationship, the in vitro release profiles of the model drug theophylline were measured for the 14 formula- tions listed in Table 1. In all cases of the coating formulations, the release profiles were measured for $5 \mathrm{~h}$ time period and the frequency of data collection was the same. To find out whether the drug release from the tablet coatings provides a zero-order release kinetics, each data set was fitted to a linear equation. The quality of the fitted model is determined by the coefficient of determination, $R^{2}$, and it is defined as:

$R^{2}=\frac{\sigma_{y}-\hat{\sigma}_{y}}{\sigma_{y}}$

where

$\sigma_{y}=\frac{1}{n} \sum_{i=1}^{n}\left(Y_{i}-\bar{Y}\right)^{2}$

and

$\hat{\sigma}_{y}=\frac{1}{n} \sum_{i=1}^{n}\left(Y_{i}-\hat{Y}_{i}\right)^{2}$

denote the sample variance and the prediction error power, respectively. Additionally, $n$ is the number of experimental data points, $Y_{i}$ the experimental observation, $\bar{Y}$, the average of the experimental data points and $\hat{Y}_{i}$ denotes the predicted value by the fitted model. The quantity $R^{2}$ lies between 0 and 1 and if the value is 1 , it can be said that the fit of the model is perfect. High $R^{2}$ values listed in Table 1 for each data set indicate that there is an excellent linear relationship between the concentration of the drug and the release time, thus, all tablet coatings prepared can provide zero-order or near-zero-order drug release. The release rates for each coating formulation were estimated from the slope of the average release profiles and they are also listed in Table 1.

The drastic change in the release rates with the composition of the coating solution is shown in Fig. 1. Within the experimental composition range covered, the highest release rate was observed in the case of the lowest cellulose acetate (P: polymer) and the highest acetone (S: solvent) concentrations in the casting 


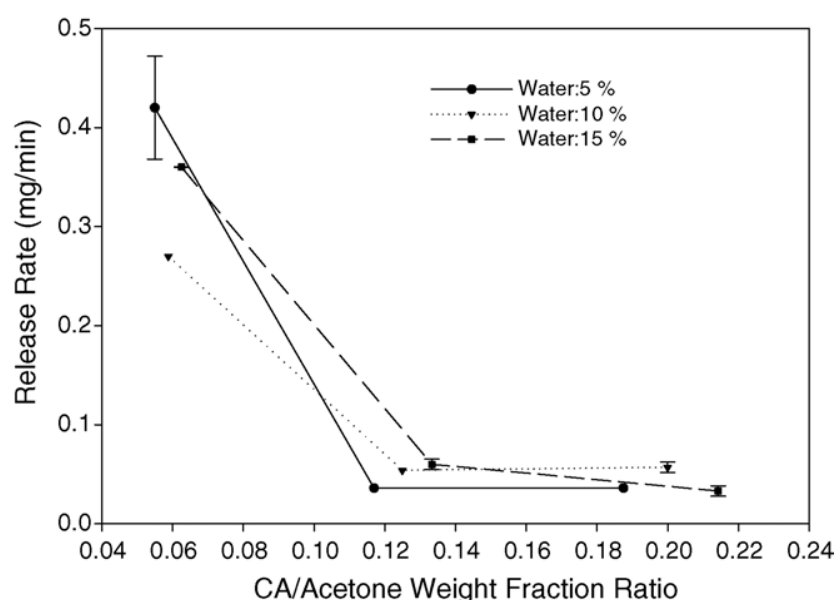

Fig. 1. Release rate of theophylline as a function of the cellulose acetate (P)/acetone (S) weight fraction ratios.

solution (polymer $(\mathrm{P}) /$ solvent $(\mathrm{S})$ weight fraction ratio $=5 / 90)$. This is caused by the final coating structure consisting of a very thin and dense top skin layer and highly porous lower sublayer as illustrated in Fig. 2. At the lowest level of polymer concentration $(5 \%)$, the thicknesses of the dense skin layers of the coatings are very small and similar to each other, thus, the structure of the lower sublayer becomes an important factor in determining the release rate of the drug. The porosity of the coating structure in the case of $90 \%$ acetone is so high that an increase in water concentration from 5 to $10 \%$ is not sufficient to produce a more porous final structure, thus, the release rate of the drug decreases. A further increase of water concentration to $15 \%$ results in an opposite effect which makes the release rate increase to a level of $0.36 \mathrm{mg} / \mathrm{min}$ due to the dominant effect of water concentration in increasing the porosity of lower sublayer.

An increment in the cellulose acetate concentration from 5 to $10 \%$ resulted in significantly lower release rates at all water concentrations because the structure of the coatings changed

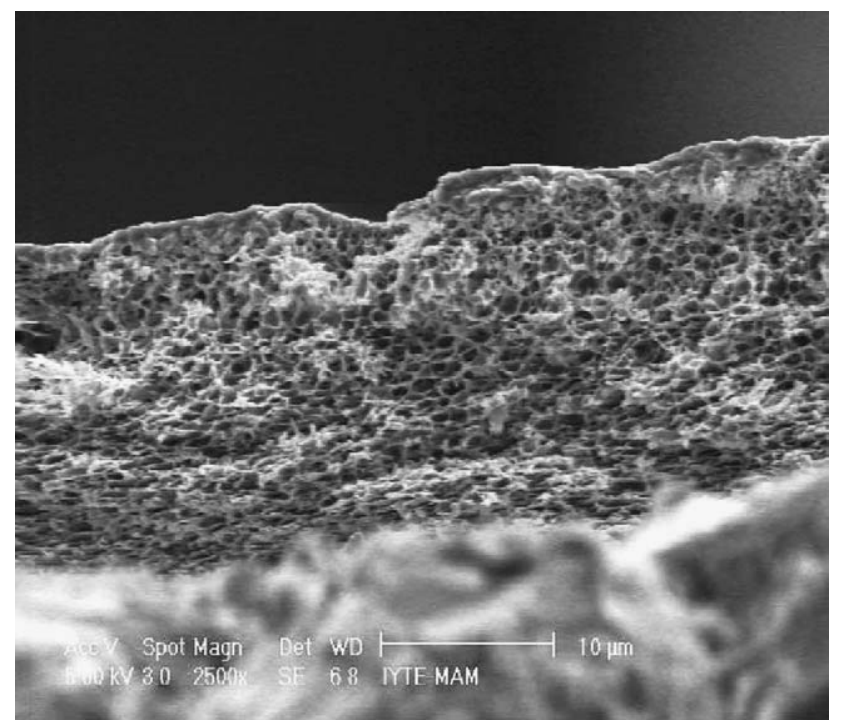

Fig. 2. SEM of the cross section of the asymmetric-membrane made with 5\% water and CA/acetone weight ratio of 5/90, magnification $2500 \times$.

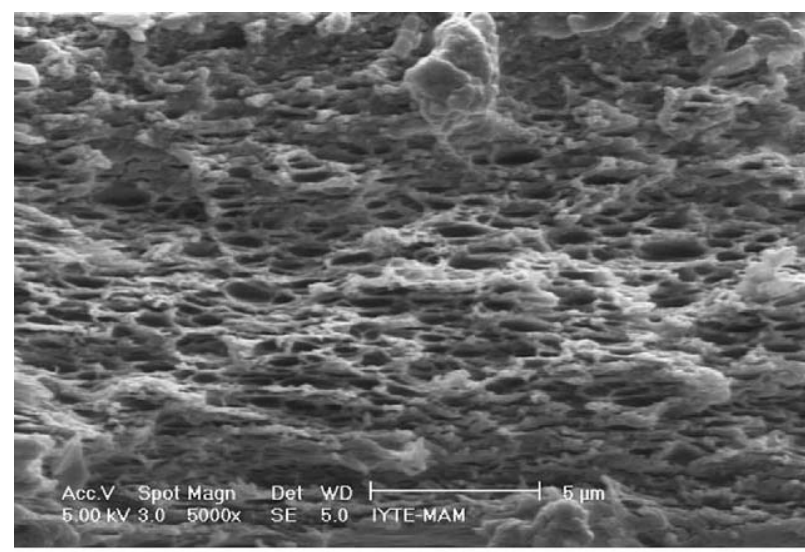

(a)

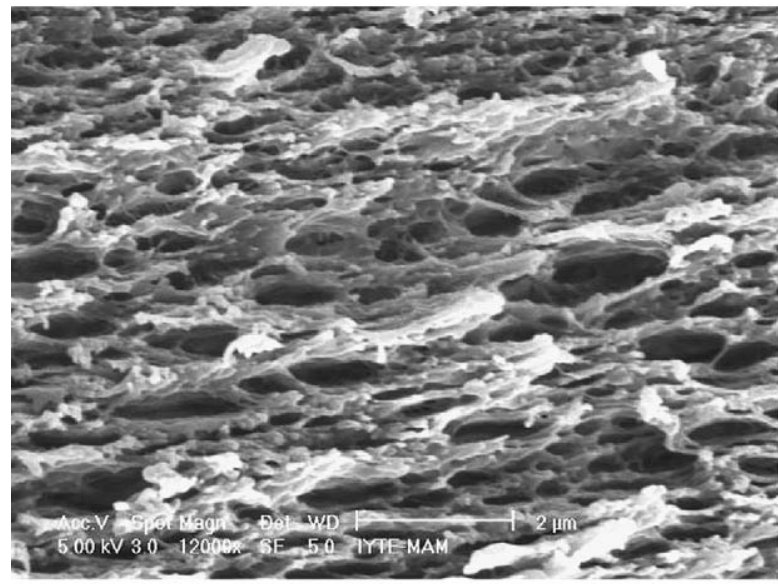

(b)

Fig. 3. SEM of the cross section of the asymmetric-membrane made with 5\% water and CA/acetone weight ratios of: (a) 10/85, magnification $5000 \times$ and (b) $15 / 80$, magnification $12,000 \times$

from porous to dense ones. Keeping the polymer concentration at $10 \%$, while changing the water concentration from 5 to $15 \%$ makes the release rates increase. This behavior is explained by the formation of more porous structures by adding more nonsolvent into the casting solution, which is in agreement with the observations of other groups $[6,16]$.

When the polymer concentration increases from 10 to $15 \%$, no significant changes in release rates were observed. As a matter of fact, in the cases of 5 and $10 \%$ water concentrations, the release rates did not change at all. This is due to unusual transport characteristics of asymmetric-membranes, which are complex functions of the properties of the different regions of the membrane. In addition to the thickness of the dense skin layer and the porosity of the lower sublayer, structural factors, such as tortuosity, pore size, shape, and connectivity of the pores also strongly affect the rate of transport through the coating. The SEM pictures taken at high magnification, which are shown in Fig. 3a and b, indicate that the tablet coating prepared with the $\mathrm{P} / \mathrm{S}$ ratio of 10/85 has a uniform and narrow pore size distribution with regular elliptic pore shapes, while the other one (P/S $15 / 80$ ) has cylindrical pores with a wide pore size distribution, forming a connected pore network. As a result, even though the resistance of dense skin layer of the coating made with $\mathrm{P} / \mathrm{S}$ ratio 


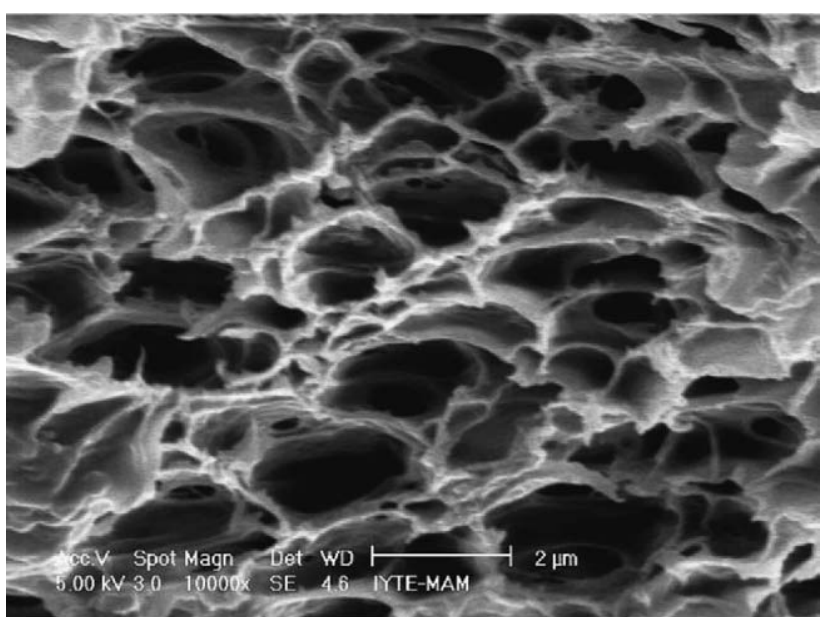

(a)

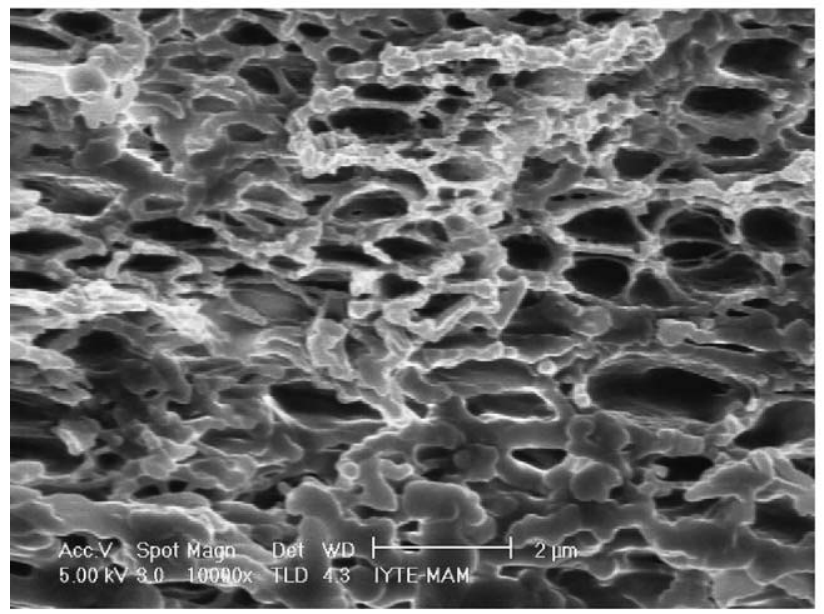

(b)

Fig. 4. SEM of the cross section of the asymmetric-membrane made with $10 \%$ water and CA/acetone weight ratios of: (a) 10/80, magnification $10,000 \times$ and (b) $15 / 75$, magnification $10,000 \times$

of $15 / 80$ is larger, its lower sublayer resistance is smaller due to the connected pore network. Consequently, the release rate of the theophylline becomes the same through both tablet coatings. The comparison of the scanning electron micrographs shown in Fig. $4 \mathrm{a}$ and $\mathrm{b}$ indicates that the tablet coating made with the P/S ratio of 10/80 has a uniform pore size distribution, cylindrical pore shapes and high tortuosity, while the tablet coating prepared with the $\mathrm{P} / \mathrm{S}$ ratio of $15 / 75$ has elliptic, irregular pore shapes and pores are isolated. Thus, the lower sublayer resistance of the coating made with the P/S ratio of 10/80 is larger due to the relatively higher tortuosity resulting in the same release rate with the coating prepared with the P/S ratio of $15 / 75$.

At the $15 \%$ polymer concentration level, the release rate increases with the change in water concentration from 5 to $10 \%$, which is mainly caused by the increased porosity. However, a further 5\% increment in the water concentration (to 15\%) made the release rate decrease back to the same level as in the case of $5 \%$ water concentration. Even though higher water concentration favors forming a more porous structure and a concomitant higher release rate, the acetone level which decreased below a critical value distructed this mechanism.

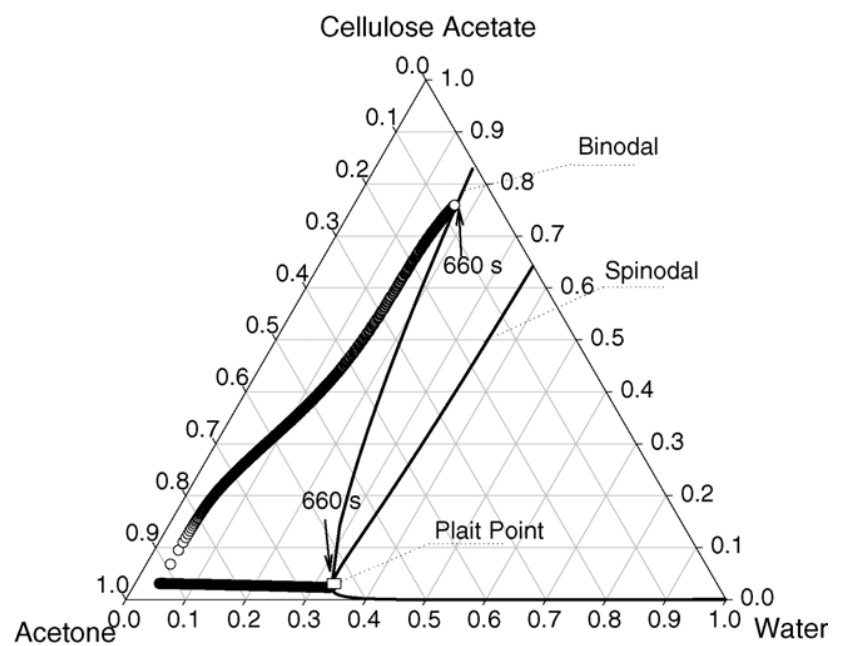

Fig. 5. Ternary phase diagram and concentration paths for the coating made with $5 \%$ cellulose acetate (CA) dissolved in $90 \%$ acetone and $5 \%$ water.

The results of dissolution studies along with the morphological observations clearly indicate that the drug release rate is strongly influenced by the morphology of the membrane. Thus, if one wishes to control the drug release characteristics of the delivery system, a quantitative understanding of the dynamics and morphology of the phase-inversion process is required. The dynamics of the phase-inversion process can be quantified in terms of the ternary phase diagram coupled with the heat and mass transfer model equations. We have obtained information about the structure of the tablet coating by plotting the composition paths on the ternary phase diagram and the polymer concentration profile at the moment of precipitation. As an illustration in Fig. 5, concentration paths in time for the tablet coating prepared with $5 \%$ cellulose acetate, $90 \%$ acetone and $5 \%$ water are shown. According to this plot, the phase separation takes place since the concentration paths in time for the drug tablet/coating and the coating/air interface cross the binodal curve at the same time. In addition, the coating/air interface enters the phase envelope at a polymer volume fraction of 0.76 , while the tablet/coating interface enters with a volume fraction of 0.023 . These two observations imply that the coating will be porous and the asymmetric in which the top layer is more dense than the lower sublayer, which was confirmed with the SEM picture shown previously in Fig. 2. The predictions have shown that phase separation was achieved for all coating formulations supporting the morphological observations and the precipitation times calculated for each case are listed in Table 1. Model predictions can also be used to determine a rough thickness of the high polymer concentration region near the coating/air interface and the pore distribution of the sublayer structure when the polymer concentration profiles at the moment of phase separation are plotted. As an illustration, such a plot is shown in Fig. 6 for coatings prepared with $10 \%$ cellulose acetate in the casting solution. Examination of these profiles leads to the following conclusions regarding the effect of increased water concentration: (1) the polymer concentration at the coating/air interface slightly decreases and (2) more uniform porosity distribution throughout the lower sublayer is favored. Also, porosity increases which 


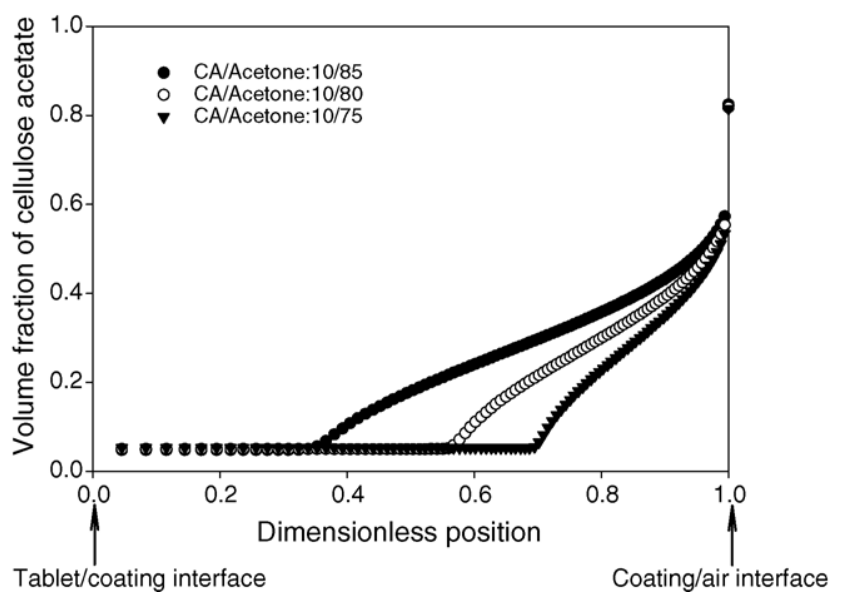

Fig. 6. Concentration profile of cellulose acetate in the membrane at the moment of precipitation for different cellulose acetate to acetone weight fraction ratios.

is in complete agreement with our release studies and the observations of other groups $[6,16]$.

\subsection{Effect of evaporation condition}

Previous studies on more traditional applications have investigated the effect of increased air velocity, i.e., forced convection conditions for a combination of dry/wet phase-inversion techniques to produce defect free, ultrahigh flux asymmetricmembranes with ultrathin skin layers [28-30]. To investigate the effect of evaporation condition on the release rate of drug, tablets were dip coated with cellulose acetate solution containing $10 \%$ cellulose acetate, $80 \%$ acetone, and $10 \%$ water and allowed to dry by blowing air across the surface with a blower (forced convection). As a comparison, tablets coated with the same solution were air-dried under natural free convection conditions.

As illustrated in Fig. 7, the release profiles of both tablet coatings show a linear behavior only at small times, and then

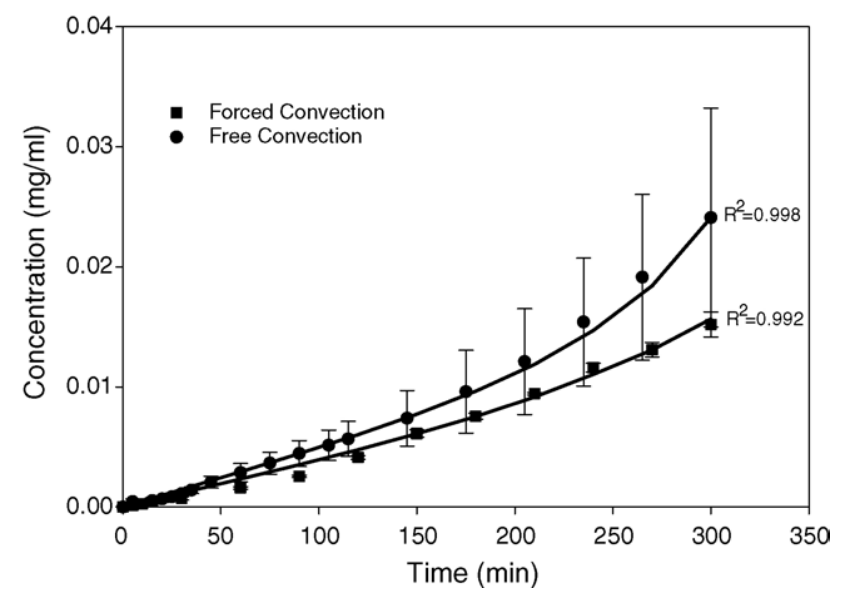

Fig. 7. Release of theophylline from tablet coatings made with $10 \%$ CA dissolved in $80 \%$ acetone and $10 \%$ water. Coated tablets were dried under free and forced convection conditions. The lines correspond to prediction from Eq. (5) using $k_{\mathrm{o}}=1.92 \times 10^{-5} \mathrm{mg} /(\mathrm{ml} \mathrm{min}) ; b_{0}=0.1179 \mathrm{ml} /(\mathrm{mg} \mathrm{min})$ for forced convection and $k_{\mathrm{o}}=2.41 \times 10^{-5} \mathrm{mg} /(\mathrm{ml} \mathrm{min}) ; b_{\mathrm{o}}=0.1174 \mathrm{ml} /(\mathrm{mg} \mathrm{min})$ for free convection. exponential increases in concentrations were observed. Based on this behavior, the release profiles were fitted to an empirical equation as:

$C=k_{\mathrm{o}} t\left[1+e^{b_{\mathrm{o}} C t}\right]$

where $k_{\mathrm{o}}$ and $b_{\mathrm{o}}$ are fitting parameters. The accuracy of Eq. (5) for correlating the release rate data in Fig. 7 was confirmed by high $R^{2}$ values, very close to 1 in both cases. Due to the presence of the second term in Eq. (5), the release rate of the drug, $\frac{\mathrm{d} C}{\mathrm{~d} t}$, is no longer constant and its dependency on concentration of drug in the dissolution medium, $C$, can be expressed as follows:

$\frac{\mathrm{d} C}{\mathrm{~d} t}=\frac{k_{\mathrm{o}}\left[1+e^{b_{\mathrm{o}} C t}\left\{1+b_{\mathrm{o}} C t\right\}\right]}{1-k_{\mathrm{o}} b_{\mathrm{o}} t^{2} e^{b_{\mathrm{o}} C t}}$

Using Eq. (6), the release rate of theophylline from the tablets dried under forced and free convection conditions were determined as 0.047 and $0.078 \mathrm{mg} / \mathrm{min}$, respectively. It should be noted that both of these values correspond to the arithmetic average of the release rates calculated at each average concentration level shown in Fig. 7. The difference in release rates can be explained by comparing the scanning electron micrographs shown in Fig. 8a and b. It can be seen that the cross sectional morphology of the tablet coating dried under forced convection condition is dense and nonporous, while porous and asymmetric structure is observed for the tablet coating dried under free convection conditions. In the dense coating, diffusional resistance to transport of the drug occurs through the overall thickness and is larger than that in the asymmetric porous coating, hence, a lower drug release rate is observed. To understand the effect of air velocity on the formation of the coating structure, we have utilized our model predictions. The composition paths plotted in Fig. 9 indicate that when the speed of air in the drying atmosphere is significantly increased, the rate of evaporation of solvent (acetone) increases dramatically and within a short time, its concentration at the surface drops to zero. This situation leads to very strong diffusional resistance within the membrane solution, thus, slow evaporation of the nonsolvent (water). Consequently, phase separation is never achieved and the resulting membrane structure becomes dense as supported by the SEM picture shown in Fig. 8a.

\subsection{Statistical analysis of experimental design}

The effect of the composition of the coating solution on the release rate of drug was investigated in detail with the 14 formulations listed in Table 1 . The drug release rate was chosen as an appropriate response variable since zero-order release was easily achieved for all tablet coatings prepared. The data in Table 1 were best fit by a special cubic equation as shown below:

release rate $=70.94 \omega_{1}+1.698 \omega_{2}+37.55 \omega_{3}-94.66 \omega_{1} \omega_{2}$

$$
-354.3 \omega_{1} \omega_{3}-49.3 \omega_{2} \omega_{3}+357.66 \omega_{1} \omega_{1} \omega_{3}
$$

The results of regression analysis are given in Table 2. An excellent fit of the experimental release rate data to Eq. (7) was 


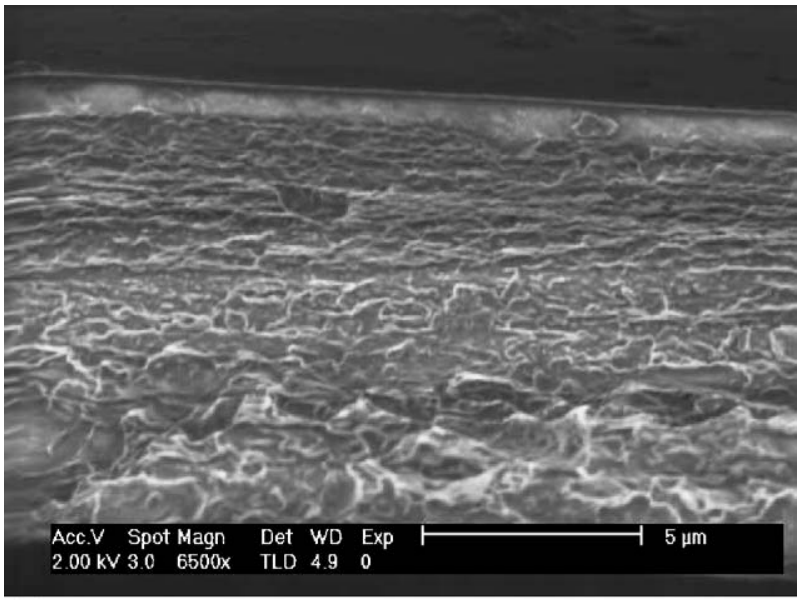

(a)

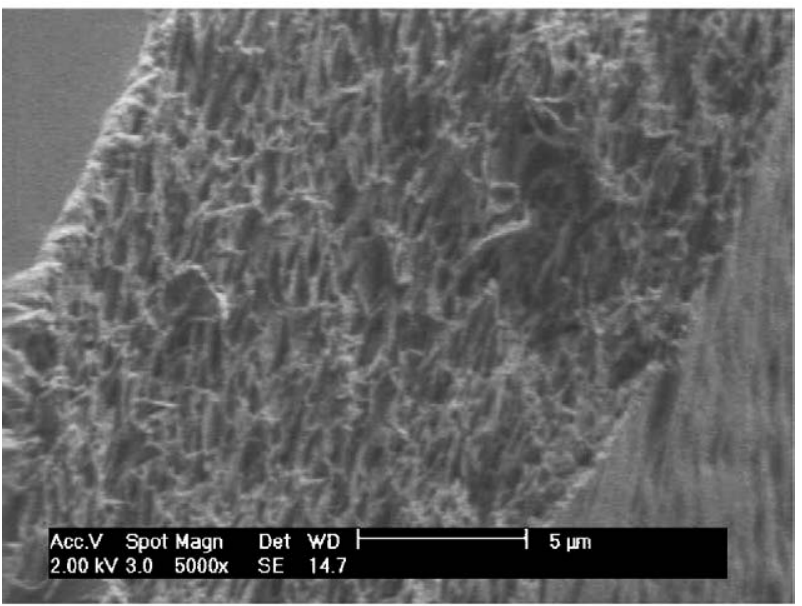

(b)

Fig. 8. SEM of the cross section of the asymmetric-membrane made with $10 \%$ CA dissolved in $80 \%$ acetone and $10 \%$ water: (a) coating solution was dried under forced convection condition, magnification $6500 \times$ and (b) coating solution was dried under free convection condition, magnification $5000 \times$.

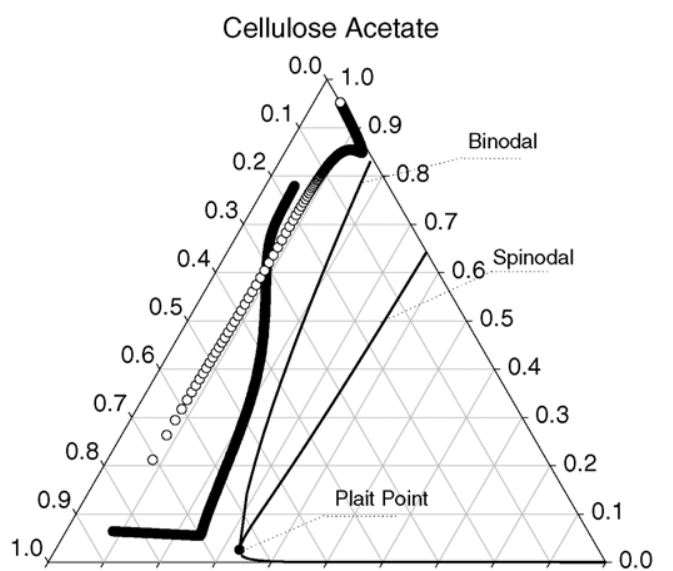

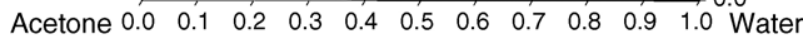

Fig. 9. Ternary phase diagram and concentration paths for the coating made with $10 \%$ CA dissolved in $80 \%$ acetone and $10 \%$ water. Coating solution was dried under forced convection condition.
Table 2

Statistical analysis of the release rate data

\begin{tabular}{|c|c|c|c|c|c|c|}
\hline \multirow[t]{2}{*}{ Model number } & \multirow[t]{2}{*}{$R^{2}$} & \multirow[t]{2}{*}{$F$ values } & \multicolumn{2}{|c|}{$\alpha=0.05$} & \multicolumn{2}{|c|}{$\alpha=0.01$} \\
\hline & & & $F_{\text {critic }^{\mathrm{a}}}$ & $\Delta F$ & $F_{\text {critic }^{a}}{ }^{a}$ & $\Delta F$ \\
\hline 1 (Full model) & 0.9801 & 42.21 & 4.21 & 38 & 8.26 & 33.95 \\
\hline $2\left(\omega_{1} \omega_{2} \omega_{3}\right)$ & 0.955 & 25.13 & 3.87 & 21.26 & 7.19 & 17.94 \\
\hline $3\left(\omega_{2} \omega_{3}\right)$ & 0.953 & 23.57 & 3.87 & 19.7 & 7.19 & 16.38 \\
\hline $4\left(\omega_{1} \omega_{3}\right)$ & 0.946 & 20.64 & 3.87 & 16.77 & 7.19 & 13.45 \\
\hline $5\left(\omega_{1} \omega_{2}\right)$ & 0.881 & 8.65 & 3.87 & 4.78 & 7.19 & 1.46 \\
\hline
\end{tabular}

${ }^{a}$ Determined from the statistical tables [32].

confirmed by the high $R^{2}$ value of 0.9801 . In addition to the $R^{2}$ values, the significance of Eq. (7) and each term in it to the prediction of the release rate of theophylline was evaluated by the $F$ statistic or $F$ value. The $F$ statistic is viewed as a ratio that expresses variance explained by the model divided by variance due to model error or experimental error and it is defined as follows:

$F=\frac{R^{2} / k}{\left(1-R^{2}\right) /(n-k-1)}$

where $k$ is the number of variables in the model. Usually, the computed value of $F$ is compared with the critical $F$ value, $F_{k, n-k, 1-\alpha}$, where $\alpha$ is a preselected significance level. If the value of $F$ is substantially greater than the critical $F$ value $\left(F_{\text {critic }}\right)$, i.e., if $\Delta F$, the difference between $F$ and $F_{\text {critic }}$ is large, then the regression equation is considered as useful in predicting the response. We have assessed the contribution of each interaction term by comparing the change in $\Delta F$ and $R^{2}$ values between the full model given in Eq. (7) and reduced models. The reduced models were obtained by deleting a specific interaction term in the full model, e.g., model 2 includes all terms in Eq. (7) except the term involving $\omega_{1} \omega_{2} \omega_{3}$. The results in Table 2 indicate that all binary and ternary interaction terms in the full model are needed for accurate prediction of the release rate since the largest $\Delta F$ values are calculated for the full model for both significance levels $\alpha=0.05$ and 0.01 . According to the criterion mentioned above, among all interactions, CA-acetone $\left(\omega_{1} \omega_{2}\right)$ was identified as the most influential factor on the response since the largest decrease in both $\Delta F$ and $R^{2}$ values compared to those of the full model were observed when the term $\omega_{1} \omega_{2}$ was deleted from the full model. This simply implies that changing the CA:acetone ratio in the coating formulation has the most significant effect on the release rate. Specifically, increasing the ratio of CA to acetone from 5/90 to $15 / 80$ resulted in a decrease of the release rate from 0.45 to $0.036 \mathrm{mg} / \mathrm{min}$ since the porosity of the membrane decreases and the thickness of the dense skin layer increases. The ratio of the composition of CA to water $\left(\omega_{1} \omega_{3}\right)$ was also found to be an important parameter on the release rate of drug as indicated by the second largest decrease in $R^{2}$ and $\Delta F$ values compared to those of the full model. Decreasing this ratio from $15 / 5$ to $5 / 15$ caused an increase in the release rate by a factor of 10 since the thickness of the dense skin layer significantly decreases. Based on the decrease in $\Delta F$ and $R^{2}$ values from those of the full model given in Table 2, the relative importance of each interaction term can be ranked as follows: $\omega_{1} \omega_{2}>\omega_{1} \omega_{3}>\omega_{2} \omega_{3}>\omega_{1} \omega_{2} \omega_{3}$. 
In order to validate the predictive capability of the empirical expression, two formulations denoted as $\mathrm{A} 1 \quad\left(\omega_{1}=7.5\right.$; $\left.\omega_{2}=82.5 ; \omega_{3}=10\right)$ and A2 $\left(\omega_{1}=10 ; \omega_{2}=82.5 ; \omega_{3}=7.5\right)$ were selected randomly from the experimental design region. Experimental release rates $\left(E_{\mathrm{RR}}\right)$ determined from the slope of the release profiles, $R^{2}$ values for the release profiles and corresponding rates predicted from Eq. (7) $\left(P_{\mathrm{RR}}\right)$ are as follows-formulation A1: $E_{\mathrm{RR}}=0.09 \mathrm{mg} / \mathrm{min} ; R^{2}=0.9816$; $P_{\mathrm{RR}}=0.125 \mathrm{mg} / \mathrm{min}$; formulation $\mathrm{A} 2: E_{\mathrm{RR}}=0.027 \mathrm{mg} / \mathrm{min}$; $R^{2}=0.9858 ; P_{\mathrm{RR}}=0.029 \mathrm{mg} / \mathrm{min}$. According to the results, the maximum absolute percentage difference between the experimental and predicted release rates is $3.5 \%$. This value lies within the residuals obtained in deriving Eq. (7). Based on this comparison, it is fair to conclude that the empirical expression derived in this study can be used as a tool to predict the release rate for any composition within the experimental design region.

\section{Conclusions}

In this study, the effects of coating solution and the evaporation condition on the phase-inversion dynamics, the ultimate coating structure and subsequent in vitro drug release properties have been examined. Our results indicate that drug release from the asymmetric-membrane based system is primarily governed by the dynamics of the phase-inversion leading to the formation of a thin outer layer and a porous sublayer. As expected, the composition of the coating solution was found to have a significant effect on the morphology of the membrane, which in turn affects the release rate of the drug from the tablets. On the other hand, the evaporation condition also plays an important role on the phase-inversion dynamics and the morphology of the final coating. By changing the velocity of air blown across wet coated tablet surface, it is possible to produce structures ranging from dense to highly porous and asymmetric leading to manipulate the release rate of the drug. Not only the velocity of air but also the role of other external environmental conditions, such as evaporation temperature and relative humidity on the phase-inversion dynamics cannot be ignored [31].

To describe the release rate of theophylline as a function of the composition of the coating solution, an empirical expression was derived from a statistical design of the experiments. Statistical analysis of the model terms in the expression allowed to identify the influence of composition of each component in the ternary coating solution on the release rate of the drug. According to this analysis, changing the $\mathrm{CA}$ :acetone ratio has the most influential effect on the release rate. Specifically, increasing the polymer content in the solution causes a slower drug release rate due to formation of a more dense morphology. Similarly, by tailoring the CA:water ratios, it is possible to effect morphological transformations from more dense to porous, thus, increasing the release rates.

Finally, it can be concluded that release kinetics of a drug from an asymmetric-membrane based drug delivery system are a function of the morphology of the membrane, which is strongly influenced by the dynamics of the phase-inversion process. Thus, these type of tablet coatings could provide improved drug deliv- ery formulations capable of releasing drug at a constant and desired rates.

\section{Acknowledgements}

The authors thank Dr. Sedat Akkurt of Izmir Institute of Technology for helping them with the experimental design, Dr. Yesim Karasulu of Ege University, Department of Pharmaceutical Technology, for useful discussions and Eczacibasi A.S. for supplying model drug theophylline.

\section{References}

[1] S. Narisawa, M. Nagata, T. Ito, H. Yoshino, Y. Hirakawa, K. Noda, Drug release behavior in gastrointestinal track of beagle dogs from multiple unit type rate-controlled or time-controlled release preparations coated with insoluble polymer-based film, J. Control. Release 33 (1995) 253.

[2] K. Thoma, K. Bechtold, Influence of aqueous coatings on the stability of enteric coated pellets and tablets, Eur. J. Pharm. Biopharm. 47 (1999) 39.

[3] M.A. Khan, S.V. Sastry, S.R. Vaithiyalingam, V. Agarwal, S. Nazzal, I.K. Reddy, Captopril gastrointestinal therapeutic system coated with cellulose acetate pseudolatex: evaluation of main effects of several formulation variables, Int. J. Pharm. 193 (2000) 147.

[4] L. Liu, J. Ku, G. Khang, B. Lee, J.M. Rhee, H.B. Lee, Nifedipine controlled delivery by sandwiched osmotic tablet system, J. Control. Release 68 (2000) 145.

[5] L. Liu, G. Khang, J.M. Rhee, H.B. Lee, Monolithic osmotic tablet system for nifedipine delivery, J. Control. Release 67 (2000) 309.

[6] S.M. Herbig, J.R. Cardinal, R.W. Korsmeyer, K.L. Smith, Asymmetricmembrane tablet coatings for osmotic drug delivery, J. Control. Release 35 (1995) 127.

[7] A.G. Thombre, J.R. Cardinal, A.R. DeNoto, S.M. Herbig, K.L. Smith, Asymmetric-membrane capsules for osmotic drug delivery. Part I: development of a manufacturing process, J. Control. Release 57 (1999) 55.

[8] A.G. Thombre, J.R. Cardinal, A.R. DeNoto, D.C. Gibbes, Asymmetricmembrane capsules for osmotic drug delivery. Part II: in vitro and in vivo drug release performance, J. Control. Release 57 (1999) 65.

[9] G.M. Zentner, G.S. Rork, K.J. Himmelstein, The controlled-porosity osmotic pump, J. Control. Release 1 (1985) 269.

[10] R.W. Baker, J.W. Brooke, Pharmaceutical drug delivery system, U.S. Patent No. 4,687,660, 1987.

[11] F. Theeuwes, A.D. Ayer, Osmotic device having composite walls, U.S. Patent No. 4,077,407, 1978.

[12] F. Theeuwes, Microporous-semipermeable laminated osmotic system, U.S. Patent No. 4,256,108, 1978.

[13] A.G. Thombre, A.R. DeNoto, D.C. Gibbes, Delivery of glipizide from asymmetric-membrane capsules using encapsulated excipients, J. Control. Release 60 (1999) 333.

[14] J.R. Cardinal, S.M. Herbig, R.W. Korsmeyer, J. Lo, K.L. Smith, G. Avinash, Use of asymmetric-membranes in delivery devices, U.S. Patent No. 5,612,059, 1997.

[15] D. Prabakaran, P. Singh, K.S. Jaganathan, S.P. Vyas, Osmotically regulated asymmetric capsular systems for simultaneous sustained delivery of anti-tubercular drugs, J. Control. Release 95 (2004) 239.

[16] D.-M. Wang, F.-C. Lin, L.-Y. Chen, J.-Y. Lai, Application of asymmetric TPX membranes to transdermal delivery of nitroglycerin, J. Control. Release 50 (1998) 187.

[17] Y.-K. Lin, H.-O. Ho, Investigations on the drug releasing mechanism from an asymmetric-membrane-coated capsule with an in situ formed delivery orifice, J. Control. Release 89 (2003) 57.

[18] S.A. Altinkaya, B. Ozbas, Modeling of asymmetric-membrane formation by dry-casting method, J. Membr. Sci. 230 (2004) 71.

[19] D.C. Montgomery, Design and Analysis of Experiments, fifth ed., John Wiley \& Sons Inc., New York, 2001. 
[20] J.-Y. Lai, F.-C. Lin, C.-C. Wang, D.-M. Wang, Effect of nonsolvent additives on the porosity and morphology of asymmetric TPX membranes, J. Membr. Sci. 118 (1996) 49.

[21] K.W. Broadhead, P.A. Tresco, Effects of fabrication conditions on the structure and function of membranes formed from poly(acrylonitrilevinylchloride), J. Membr. Sci. 147 (1998) 235.

[22] T.-H. Young, D.-J. Lin, J.-J. Gau, W.-Y. Chuang, L.-P. Cheng, Morphology of crystalline Nylon-610 membranes prepared by the immersion-precipitation process: competition between crystallization and liquid-liquid phase separation, Polymer 40 (1999) 5011.

[23] J. Won, H.C. Park, U.Y. Kim, Y.S. Kang, S.H. Yoo, J.Y. Jho, The effect of dope solution characteristics on the membrane morphology and gas transport properties: PES/-BL/NMP system, J. Membr. Sci. 162 (1999) 247.

[24] J. Won, H.J. Lee, Y.S. Kang, The effect of dope solution characteristics on the membrane morphology and gas transport properties. Part 2: PES/BL system, J. Membr. Sci. 176 (2000) 11.

[25] W.-Y. Chuang, T.-H. Young, W.-Y. Chiu, C.-Y. Lin, The effect of polymeric additives on the structure and permeability of poly(vinyl alcohol) asymmetric-membranes, Polymer 41 (2000) 5633.

[26] S.-C. Fan, Y.-C. Wang, C.-L. Li, K.-R. Lee, D.-J. Liaw, H.-P. Huang, J.-Y. Lai, Effect of coagulation media on membrane formation and vapor permeation performance of novel aromatic polyamide membrane, J. Membr. Sci. 204 (2002) 67.

[27] N.A. Mohamed, A.O.H. Al-Dossary, Structure-property relationships for novel wholly aromatic polyamide-hydrazides containing various proportions of para-phenylene and meta-phenylene units. Part III: preparation and properties of semi-permeable membranes for water desalination by reverse osmosis separation performance, Eur. Polym. J. 39 (2003) 1653.

[28] I. Pinnau, W. Koros, Structures and gas separation property asymmetric polysulfone membranes made by dry, wet, and dry/wet phase-inversion, J. Appl. Polym. Sci. 43 (1991) 1491.

[29] I.D. Sharpe, A.F. Ismail, S.J. Shilton, A study of extrusion shear and forced convection residence time in the spinning of polysulfone hollow fiber membranes for gas separation, Sep. Purif. Technol. 17 (1999) 101.

[30] A.F. Ismail, B.C. Ng, W.A.W. Abdul Rahman, Effects of shear rate and forced convection residence time on asymmetric polysulfone membranes structure and gas separation performance, Sep. Purif. Technol. 33 (2003) 255.

[31] S.A. Altınkaya, H. Yenal, B. Ozbas, Membrane formation by dry-cast process: model validation through morphological studies, J. Membr. Sci. 249 (2005) 163.

[32] D.G. Kleinbaum, L.L. Kupper, K.E. Muller, Applied Regression Analysis and Other Multivariable Methods, second ed., Duxbury Press, Belmont, 1987. 